\title{
The Inertial Manifolds for a Class of Higher-Order Coupled Kirchhoff-Type Equations
}

\author{
Guoguang Lin, Sanmei Yang \\ Department of Mathematics, Yunnan University, Kunming, China \\ Email: gglin@ynu.edu.cn, 1934211371@qq.com
}

How to cite this paper: Lin, G.G. and Yang, S.M. (2018) The Inertial Manifolds for a Class of Higher-Order Coupled Kirchhoff-Type Equations. Journal of Applied Mathematics and Physics, 6, 1055-1064. https://doi.org/10.4236/jamp.2018.65091

Received: April 7, 2018

Accepted: May 27, 2018

Published: May 30, 2018

Copyright $\odot 2018$ by authors and Scientific Research Publishing Inc. This work is licensed under the Creative Commons Attribution International License (CC BY 4.0).

http://creativecommons.org/licenses/by/4.0/

\begin{abstract}
In this paper, we mainly deal with a class of higher-order coupled Kirchhoff-type equations. At first, we take advantage of Hadamard's graph to get the equivalent form of the original equations. Then, the inertial manifolds are proved by using spectral gap condition. The main result we gained is that the inertial manifolds are established under the proper assumptions of $M(s)$ and $g_{i}(u, v), i=1,2$.
\end{abstract}

\section{Keywords}

Higher-Order Coupled Kirchhoff-Type Equations, Inertial Manifold, Hadamard's Graph, Spectral Gap Condition

\section{Introduction}

This paper mainly deals with existence of inertial manifolds for a class of higher-order coupled Kirchhoff-type equations:

$$
\begin{gathered}
u_{t t}+M\left(\|\nabla u\|^{2}+\|\nabla v\|^{2}\right)(-\Delta)^{m} u+\beta(-\Delta)^{m} u_{t}+g_{1}(u, v)=f_{1}(x), \\
v_{t t}+M\left(\|\nabla u\|^{2}+\|\nabla v\|^{2}\right)(-\Delta)^{m} v+\beta(-\Delta)^{m} v_{t}+g_{2}(u, v)=f_{2}(x), \\
u(x, 0)=u_{0}(x), u_{t}(x, 0)=u_{1}(x), x \in \Omega, \\
v(x, 0)=v_{0}(x), v_{t}(x, 0)=v_{1}(x), x \in \Omega, \\
\left.u\right|_{\partial \Omega}=0,\left.\frac{\partial^{i} u}{\partial \mu^{i}}\right|_{\partial \Omega}=0, i=1,2,3, \cdots, m-1, \\
\left.v\right|_{\partial \Omega}=0,\left.\frac{\partial^{i} v}{\partial v^{i}}\right|_{\partial \Omega}=0, i=1,2,3, \cdots, m-1,
\end{gathered}
$$


where $m>1$ is an integer constant, $\Omega$ is a bounded domain of $R^{n}$ with a smooth Dirichlet boundary $\partial \Omega, u_{0}(x), u_{1}(x), v_{0}(x), v_{1}(x)$ are the initial value. $\mu_{i}$ and $v_{i}$ are the unit outward normal on $\partial \Omega, M(s)$ is a nonnegative $C^{1}$ function, $(-\Delta)^{m} u_{t}$ and $(-\Delta)^{m} v_{t}$ are strongly damping, $g_{1}(u, v)$ and $g_{2}(u, v)$ are nonlinear source terms, $f_{1}(x)$ and $f_{2}(x)$ are given forcing functions.

It is significant to establish inertial manifolds for the study of the long-time behavior of infinite dimensional dynamical systems, because it is an important bridge between infinite-dimensional dynamic system and finite-dimensional dynamical system.

To better carry out our work, let's recall some results regarding wave equations.

Jingzhu $\mathrm{Wu}$ and Guoguang Lin [1] studied the following two-dimensional strong damping Boussinesq equation while $\alpha>2$ :

$$
\begin{gathered}
u_{t t}-\alpha \Delta u_{t}-\Delta u+u^{2 k+1}=f(x, y),(x, y) \in \Omega, \\
u(x, y, 0)=u_{0}(x, y),(x, y) \in \Omega, \\
u(x, y, t)=u(x+\pi, y, t)=u(x, y+\pi, t)=0,(x, y) \in \Omega,
\end{gathered}
$$

where $\Omega=(0, \pi) \times(0, \pi) \subset R \times R, t>0$. They obtained result that is existence of inertial manifolds.

Guigui Xu, Libo Wang and Guoguang Lin [2] investigated the strongly damped wave equation:

$$
\begin{gathered}
u_{t t}-\alpha \Delta u+\beta \Delta^{2} u-\gamma \Delta u_{t}+g(u)=f(x, t),(x, t) \in \Omega \times R^{+}, \\
u(x, 0)=u_{0}(x), u_{t}(x, 0)=u_{1}(x), x \in \Omega \\
\left.u\right|_{\partial \Omega}=0,\left.\Delta u\right|_{\partial \Omega}=0,(x, t) \in \partial \Omega \times R^{+}
\end{gathered}
$$

They gave some assumptions for the nonlinearity term $g(u)$ to satisfy the following inequalities:

(A1) $\lim _{|s| \rightarrow \infty} \inf \frac{G(s)}{s^{2}} \geq 0, s \in R, G(s)=\int_{0}^{s} g(r) \mathrm{d} r$.

(A2) There is positive constant $C_{1}$ such that $\lim _{|s| \rightarrow \infty} \inf \frac{s g(s)-C_{1} G(s)}{s^{2}} \geq 0, s \in R$.

According to the above assumptions, they proved the inertial manifolds by using the Hadamard's graph transformation method.

Ruijin Lou, Penhui Lv, Guoguang Lin [3] considered a class of generalized nonlinear Kirchhoff-Sine-Gordon equation:

$$
\begin{gathered}
u_{t t}-\beta \Delta u_{t}+\alpha u_{t}-\phi\left(\|\nabla u\|^{2}\right) \Delta u+g(\sin u)=f(x), \\
u(x, t)=0, x \in \Omega, t \geq 0 \\
u(x, 0)=u_{0}(x), u_{t}(x, 0)=u_{1}(x), x \in \Omega .
\end{gathered}
$$

Under some reasonable assumptions, they obtained some results that are 
squeezing property of the nonlinear semigroup associated with this equation and the existence of exponential attractors and inertial manifolds.

Lin Chen, Wei Wang and Guoguang Lin [4] studied higher-order Kirchhoff-type equation with nonlinear strong dissipation in $n$ dimensional space:

$$
\begin{gathered}
u_{t t}+(-\Delta)^{m} u_{t}+\phi\left(\|\nabla u\|^{2}\right)(-\Delta)^{m} u+g(u)=f(x), x \in \Omega, t>0, m>1, \\
u(x, t)=0, \frac{\partial^{i} u}{\partial v^{i}}=0, i=1,2, \cdots, m-1, x \in \partial \Omega, t>0, \\
u(x, 0)=u_{0}(x), u_{t}(x, 0)=u_{1}(x) .
\end{gathered}
$$

For the above equation, they made some suitable assumptions about $\phi(s)$ and $g(u)$ to get existence of exponential attractors and inertial manifolds.

In this article, we first take advantage of Hadamard's graph to transform equations (1.1)-(1.2) into an equivalent one-order system of form. Then, we proved the inertial manifolds by using spectral gap condition.

\section{Preliminaries}

We denote the some simple symbols, $\|\cdot\|$ and $($,$) stand for norm and inner$ product of $H$ and $H^{m}=H^{m}(\Omega), H_{0}^{m}(\Omega)=H^{m}(\Omega) \cap H_{0}^{1}(\Omega)$, $H_{0}^{2 m}(\Omega)=H^{2 m}(\Omega) \cap H_{0}^{1}(\Omega), H=L^{2}(\Omega), f_{i}=f_{i}(x),(i=1,2)$, $v=\|\nabla u\|^{2}+\|\nabla v\|^{2}, \quad v_{k}=\left\|\nabla u_{k}\right\|^{2}+\left\|\nabla v_{k}\right\|^{2},(k=1,2, \cdots),\|\cdot\|=\|\cdot\|_{L^{2}(\Omega)}$, $X=H_{0}^{m}(\Omega) \times H_{0}^{m}(\Omega) \times L^{2}(\Omega) \times L^{2}(\Omega),\|\|_{\infty}=\|\|_{L^{\infty}(\Omega)} . \quad c_{i},(i=1,2, \cdots)$ are various positive constants.

Next, we give some assumptions needed for problem (1.1)-(1.6).

$$
\begin{aligned}
& \text { (H1) } g_{i}(u, v),(i=1,2), M(s) \in C^{1}(R) . \\
& \text { (H2) } \varepsilon \leq m_{0} \leq M(s) \leq m_{1}=\frac{\beta^{2} \mu_{1}-1}{4} .
\end{aligned}
$$

Then, we give the basic concepts below.

Definition 2.1. [6] An inertial manifold $\mu$ is a finite-dimensional manifold enjoying the following three properties:

1) $\mu$ is Lipschitz,

2) $\mu$ is positively invariant for the semi-group $\{S(t)\}_{t \geq 0}$, i.e. $S(t) \mu \subset \mu, \forall t \geq 0$,

(3) $\mu$ attracts exponentially all the orbits of the solution.

Definition 2.2. [6] Let $A: X \rightarrow X$ be an operator and assume that $F \in C_{b}(X, X)$ satisfies the Lipschitz condition:

$$
\|F(U)-F(V)\|_{X} \leq l_{F}\|U-V\|_{X}, U, V \in X,
$$

where $X=H_{0}^{m}(\Omega) \times H_{0}^{m}(\Omega) \times L^{2}(\Omega) \times L^{2}(\Omega)$. The operator $A$ is said to satisfy the spectral gap condition relative to $F$, if the point spectrum of the operator $A$ can be divided into two parts $\sigma_{1}$ and $\sigma_{2}$, of which $\sigma_{1}$ is finite, and such that, if

$$
\Lambda_{1}=\sup \left\{\operatorname{Re} \lambda \mid \lambda \in \sigma_{1}\right\}, \Lambda_{2}=\sup \left\{\operatorname{Re} \lambda \mid \lambda \in \sigma_{2}\right\}
$$


and

$$
X_{i}=\operatorname{span}\left\{\omega_{j} \mid j \in \sigma_{i}\right\}, i=1,2
$$

Then

$$
\Lambda_{2}-\Lambda_{1}>4 l_{F}
$$

And the orthogonal decomposition

$$
X=X_{1} \oplus X_{2},
$$

holds with continuous orthogonal projections $P_{1}: X \rightarrow X_{1}$ and $P_{1}: X \rightarrow X_{2}$.

Lemma 2.1. [7] Let the eigenvalues $\lambda_{j}^{ \pm}, j \geq 1$ be arranged in nondecreasing order, for all $m \in N$, there is $N \geq m$ such that $\lambda_{N}^{-}$and $\lambda_{N+1}^{-}$are consecutive.

\section{Inertial Manifold}

In this section, we use the Hadamard's graph transformation method to prove the existence of inertial manifolds of problem (1.1)-(1.6) when $N$ is sufficiently large.

Equations (1.1)-(1.2) are equivalent to the following one order evolution equation:

$$
U_{t}+H U=F(U),
$$

where $U=(u, v, p, q), p=u_{t}, q=v_{t}$,

$$
\begin{gathered}
H=\left(\begin{array}{cccc}
0 & 0 & -I & 0 \\
0 & 0 & 0 & -I \\
M(v)(-\Delta)^{m} & 0 & \beta(-\Delta)^{m} & 0 \\
0 & M(v)(-\Delta)^{m} & 0 & \beta(-\Delta)^{m}
\end{array}\right), \\
F(U)=\left(\begin{array}{c}
0 \\
0 \\
f_{1}(x)-g_{1}(u, v) \\
f_{2}(x)-g_{2}(u, v)
\end{array}\right) \\
D(H)=\left\{(u, v, p, q) \in H^{2 m} \times H^{2 m} \times H^{m} \times H^{m}\right\}
\end{gathered}
$$

In $X$, we denote the usual graph norm, which is introduced by the scalar product as the following form

$$
\begin{aligned}
(U, V)_{X}= & \left(M(s) \cdot \nabla^{m} u, \nabla^{m} \bar{y}_{1}\right)+\left(M(s) \cdot \nabla^{m} v, \nabla^{m} \bar{y}_{2}\right), \\
& +\left(\bar{z}_{1}, p\right)+\left(\bar{z}_{2}, q\right)
\end{aligned}
$$

where $U=(u, v, p, q), V=\left(y_{1}, y_{2}, z_{1}, z_{2}\right) . \bar{y}_{1}, \bar{y}_{2}, \bar{z}_{1}, \bar{z}_{2}$ denote the conjugation of $y_{1}, y_{2}, z_{1}, z_{2}$ respectively, $u, v, y_{1}, y_{2} \in H_{0}^{2 m}(\Omega), p, q, z_{1}, z_{2} \in H_{0}^{m}(\Omega)$.

For $U \in D(H)$, we have

$$
\begin{aligned}
(H U, U)_{X}= & \left(-M(v) \nabla^{m} p, \nabla^{m} \bar{u}\right)+\left(-M(v) \nabla^{m} q, \nabla^{m} \bar{v}\right) \\
& +\left(\bar{p}, M(v)(-\Delta)^{m} u+\beta(-\Delta)^{m} p\right) \\
& +\left(\bar{q}, M(v)(-\Delta)^{m} v+\beta(-\Delta)^{m} q\right) \\
= & \beta\left\|\nabla^{m} p\right\|^{2}+\beta\left\|\nabla^{m} q\right\|^{2} \geq 0 .
\end{aligned}
$$


Therefore, the operator $H$ in (3.2) is monotone, and $(H U, U)_{X}$ is a nonnegative and real number.

To obtain the eigenvalues of $H$, we consider the following eigenvalue equation:

$$
H U=\lambda U, U=(u, v, p, q) \in X
$$

That is

$$
\left\{\begin{array}{l}
-p=\lambda u, \\
-q=\lambda v, \\
M(v)(-\Delta)^{m} u+\beta(-\Delta)^{m} p=\lambda p . \\
M(v)(-\Delta)^{m} v+\beta(-\Delta)^{m} q=\lambda q .
\end{array}\right.
$$

The first two equations in (3.8) are brought into the last two equations in (3.8) respectively, we get

$$
\left\{\begin{array}{l}
\lambda^{2} u+M(v)(-\Delta)^{m} u-\beta \lambda(-\Delta)^{m} u=0 \\
\lambda^{2} v+M(v)(-\Delta)^{m} v-\beta \lambda(-\Delta)^{m} v=0 \\
\left.u\right|_{\partial \Omega}=0,\left.\frac{\partial^{i} u}{\partial \mu^{i}}\right|_{\partial \Omega}=0, i=1,2,3, \cdots, m-1, \\
\left.v\right|_{\partial \Omega}=0,\left.\frac{\partial^{i} v}{\partial v^{i}}\right|_{\partial \Omega}=0, i=1,2,3, \cdots, m-1 .
\end{array}\right.
$$

Let $u_{k}$ and $v_{k}$ replace $u$ and $v$ in (3.9) respectively. And then taking $u_{k}$ and $v_{k}$ inner product respectively, we obtain

$$
\begin{aligned}
& \lambda^{2}\left\|u_{k}\right\|^{2}+M\left(v_{k}\right)\left\|\nabla^{m} u_{k}\right\|^{2}-\beta \lambda\left\|\nabla^{m} u_{k}\right\|^{2}=0 . \\
& \lambda^{2}\left\|v_{k}\right\|^{2}+M\left(v_{k}\right)\left\|\nabla^{m} v_{k}\right\|^{2}-\beta \lambda\left\|\nabla^{m} v_{k}\right\|^{2}=0 .
\end{aligned}
$$

Summing up (3.10) and (3.11), we get

$$
\lambda^{2}\left(\left\|u_{k}\right\|^{2}+\left\|v_{k}\right\|^{2}\right)+M\left(\sqrt[m]{\mu_{k}}\right)\left(\left\|\nabla^{m} u_{k}\right\|^{2}+\left\|\nabla^{m} v_{k}\right\|^{2}\right)-\beta \lambda\left(\left\|\nabla^{m} u_{k}\right\|^{2}+\left\|\nabla^{m} v_{k}\right\|^{2}\right)=0 .
$$

When (3.12) is considered an a yuan quadratic equation on $\lambda$, we can get

$$
\lambda_{k}^{ \pm}=\frac{\beta \mu_{k} \pm \sqrt{\beta^{2} \mu_{k}^{2}-4 \mu_{k} M\left(\sqrt[m]{\mu_{k}}\right)}}{2},
$$

where $\mu_{k}$ is the eigenvalue of $(-\Delta)^{m}$ in $H_{0}^{2 m}$, then $\mu_{k}=c_{0} k^{\frac{m}{n}}$. if $\beta^{2} \mu_{k} \geq 4 M\left(\sqrt[m]{\mu_{k}}\right)$, that is $\beta^{2} \mu_{k} \geq 4 m_{1}$, the eigenvalues of $H$ are all positive and real numbers, the corresponding eigenfunction have the form $U_{k}^{ \pm}=\left(u_{k}, v_{k},-\lambda_{k}^{ \pm} u_{k},-\lambda_{k}^{ \pm} v_{k}\right)$. For (3.13) and future reference, we observe that for all $k \geq 1$,

$$
\left\|\nabla^{m} u_{k}\right\|^{2}+\left\|\nabla^{m} v_{k}\right\|^{2}=\mu_{k},\left\|u_{k}\right\|^{2}+\left\|v_{k}\right\|^{2}=1,\left\|\nabla^{-m} u_{k}\right\|^{2}+\left\|\nabla^{-m} v_{k}\right\|^{2}=\frac{1}{\mu_{k}} .
$$

Lemma $3.1 g_{i}: H_{0}^{m} \times H_{0}^{m} \rightarrow H_{0}^{m} \times H_{0}^{m}, i=1,2$ is uniformly bounded and globally Lipschitz continuous. 
Proof. $\forall u_{1}, u_{2}, v_{1}, v_{2} \in H_{0}^{m}(\Omega)$, we get

$\left\|g_{i}\left(u_{1}, v_{1}\right)-g_{i}\left(u_{2}, v_{2}\right)\right\|_{H_{0}^{m} \times H_{0}^{m}} \leq\left\|g_{i u}\left(\xi, v_{1}\right)\right\|_{\infty}\left\|u_{1}-u_{2}\right\|_{H_{0}^{m}}+\left\|g_{i v}\left(u_{2}, \eta\right)\right\|_{\infty}\left\|v_{1}-v_{2}\right\|_{H_{0}^{m}}$,

where $\xi=\theta u_{1}+(1-\theta) u_{2}, \eta=\theta v_{1}+(1-\theta) v_{2}, 0<\theta<1$. According to (H1), we can obtain

$$
\begin{aligned}
& \left\|g_{i}\left(u_{1}, v_{1}\right)-g_{i}\left(u_{2}, v_{2}\right)\right\|_{H_{0}^{m} \times H_{0}^{m}} \\
& \leq C_{1}\left\|u_{1}-u_{2}\right\|_{H_{0}^{m}}+C_{2}\left\|v_{1}-v_{2}\right\|_{H_{0}^{m}} \leq \frac{l}{2}\left(\left\|u_{1}-u_{2}\right\|_{H_{0}^{m}}+\left\|v_{1}-v_{2}\right\|_{H_{0}^{m}}\right) .
\end{aligned}
$$

Theorem 3.1 If $\beta^{2} \mu_{k} \geq 4 m_{1}$ holds, $l / 2$ is maximum Lipschitz constant of $g_{i}(u, v),(i=1,2)$, and if $N_{1} \in N$ is sufficiently large such that when $N \geq N_{1}$, the following inequality holds:

$$
\left(\mu_{N+1}-\mu_{N}\right)\left(\frac{\beta}{2}-\frac{1}{2} \sqrt{\beta^{2} \mu_{k}-4 m_{1}}\right) \geq \frac{4 \sqrt{2} l}{\sqrt{\beta^{2} \mu_{k}-4 m_{1}}}+1,
$$

Then the operator $H$ satisfies the spectral gap condition of (2.6).

Proof. When $\beta^{2} \mu_{k} \geq 4 m_{1}$, all the eigenvalues of $H$ are real and positive, and we can easily know that both sequences $\left\{\lambda_{k}^{-}\right\}_{k \geq 1}$ and $\left\{\lambda_{k}^{+}\right\}_{k \geq 1}$ are increasing.

The whole process of proof is divided into four steps.

Step 1. Since $\lambda_{k}^{ \pm}$is arranged in nondecreasing order. According to Lemma 2.1, given $N$ such that $\lambda_{N}^{-}$and $\lambda_{N+1}^{-}$are consecutive, we separate the eigenvalue of $H$ as

$$
\begin{gathered}
\sigma_{1}=\left\{\lambda_{j}^{-}, \lambda_{k}^{+} \mid \max \left\{\lambda_{j}^{-}, \lambda_{k}^{+}\right\} \leq \lambda_{N}^{-}\right\}, \\
\sigma_{2}=\left\{\lambda_{j}^{-}, \lambda_{k}^{ \pm} \mid \lambda_{j}^{-} \leq \lambda_{N}^{-} \leq \min \left\{\lambda_{j}^{-}, \lambda_{k}^{ \pm}\right\}\right\},
\end{gathered}
$$

Step 2. We make decomposition of $X$

$$
\begin{aligned}
& X_{1}=\operatorname{span}\left\{U_{j}^{-}, U_{k}^{+} \mid \lambda_{j}^{-}, \lambda_{k}^{+} \in \sigma_{1}\right\} . \\
& X_{2}=\operatorname{span}\left\{U_{j}^{+}, U_{k}^{ \pm} \mid \lambda_{j}^{-}, \lambda_{k}^{+} \in \sigma_{2}\right\} .
\end{aligned}
$$

In order to make these two subspaces orthogonal and satisfy spectral inequality (2.6) $\Lambda_{1}=\lambda_{N}^{-}, \Lambda_{2}=\lambda_{N+1}^{-}$, we further decompose

$$
X_{2}=X_{c} \oplus X_{R},
$$

with

$$
\begin{gathered}
X_{c}=\operatorname{span}\left\{U_{j}^{+} \mid \lambda_{j}^{-} \leq \lambda_{N}^{-}<\lambda_{j}^{+}\right\}, \\
X_{R}=\operatorname{span}\left\{U_{R}^{ \pm} \mid \lambda_{N}^{-} \leq \lambda_{k}^{ \pm}\right\} .
\end{gathered}
$$

And let $X_{N}=X_{1} \oplus X_{c}$. Next, we stipulate an eigenvalue scale product of $X$ such that $X_{1}$ and $X_{2}$ are orthogonal, therefore we need to introduce two functions:

Let $\Phi: X_{N} \rightarrow R, \psi: X_{R} \rightarrow R$. 


$$
\begin{aligned}
\Phi(U, V)= & \beta\left(\nabla^{m} u, \nabla^{m} \bar{y}_{1}\right)+2 \beta\left(\nabla^{-m} z_{1}, \nabla^{m} u\right)+2 \beta\left(\nabla^{m} v, \nabla^{m} \bar{y}_{2}\right) \\
& +2 \beta\left(\nabla^{-m} z_{2}, \nabla^{m} v\right)+2 \beta\left(\nabla^{-m} p, \nabla^{m} \bar{y}_{1}\right)+4\left(\nabla^{-m} p, \nabla^{-m} z_{1}\right) \\
& +2 \beta\left(\nabla^{-m} q, \nabla^{m} \bar{y}_{2}\right)+4\left(\nabla^{-m} q, \nabla^{-m} z_{2}\right)-4 M(v)\left(\bar{u}, y_{1}\right) \\
& +\left(2 \beta^{2}-\beta\right)\left(\nabla^{m} \bar{u}, \nabla^{m} y_{1}\right)-4 M(v)\left(\bar{v}, y_{2}\right)+\left(2 \beta^{2}-\beta\right)\left(\nabla^{m} \bar{v}, \nabla^{m} y_{2}\right),
\end{aligned}
$$

$$
\begin{aligned}
\psi(U, V)= & \left(\nabla^{m} u, \nabla^{m} y_{1}\right)+\left(\nabla^{m} v, \nabla^{m} y_{2}\right)+2\left(\nabla^{-m} z_{1}, \nabla^{m} u\right)+2\left(\nabla^{-m} z_{2}, \nabla^{m} v\right) \\
& +\left(\nabla^{-m} z_{1}, \nabla^{-m} p\right)+\left(\nabla^{-m} z_{2}, \nabla^{-m} q\right)-4 M(v)\left(\bar{u}, y_{1}\right) \\
& +2 \beta^{2}\left(\nabla^{m} \bar{u}, \nabla^{m} y_{1}\right)-4 M(v)\left(\bar{v}, y_{2}\right)+2 \beta^{2}\left(\nabla^{m} \bar{v}, \nabla^{m} y_{2}\right),
\end{aligned}
$$

where $U=(u, v, p, q), V=\left(y_{1}, y_{2}, z_{1}, z_{2}\right) . \bar{y}_{1}, \bar{y}_{2}, \bar{z}_{1}, \bar{z}_{2}$ respectively are the conjugation of $y_{1}, y_{2}, z_{1}, z_{2}$.

Let $U=(u, v, p, q) \in X_{N}$, then

$\Phi(U, U)$

$=\beta\left(\nabla^{m} u, \nabla^{m} \bar{u}\right)+2 \beta\left(\nabla^{-m} p, \nabla^{m} u\right)+\beta\left(\nabla^{m} v, \nabla^{m} \bar{v}\right)+2 \beta\left(\nabla^{-m} q, \nabla^{m} v\right)$

$+2 \beta\left(\nabla^{-m} p, \nabla^{m} \bar{u}\right)+4\left(\nabla^{-m} p, \nabla^{-m} p\right)+2 \beta\left(\nabla^{-m} q, \nabla^{m} \bar{v}\right)$

$+4\left(\nabla^{-m} q, \nabla^{-m} q\right)-4 M(v)(\bar{u}, u)+\left(2 \beta^{2}-\beta\right)\left(\nabla^{m} \bar{u}, \nabla^{m} u\right)$

$-4 M(v)(\bar{v}, v)+\left(2 \beta^{2}-\beta\right)\left(\nabla^{m} \bar{v}, \nabla^{m} v\right)$

$=\beta\left\|\nabla^{m} u\right\|^{2}-2\left\|\nabla^{-m} p\right\|^{2}-\frac{\beta^{2}}{2}\left\|\nabla^{m} u\right\|^{2}+\beta\left\|\nabla^{m} v\right\|^{2}-2\left\|\nabla^{-m} q\right\|^{2}-\frac{\beta^{2}}{2}\left\|\nabla^{m} v\right\|^{2}$

$-2\left\|\nabla^{-m} p\right\|^{2}-\frac{\beta^{2}}{2}\left\|\nabla^{m} \bar{u}\right\|^{2}+4\left\|\nabla^{-m} p\right\|^{2}-2\left\|\nabla^{-m} q\right\|^{2}-\frac{\beta^{2}}{2}\left\|\nabla^{m} \bar{v}\right\|^{2}+4\left\|\nabla^{-m} q\right\|^{2}$

$-4 M(v)\|u\|^{2}+\left(2 \beta^{2}-\beta\right)\left\|\nabla^{m} u\right\|^{2}-4 M(v)\|v\|^{2}+\left(2 \beta^{2}-\beta\right)\left\|\nabla^{m} v\right\|^{2}$

$\geq \beta^{2}\left(\left\|\nabla^{m} u\right\|^{2}+\left\|\nabla^{m} v\right\|^{2}\right)-4 M(v)\left(\|u\|^{2}+\|v\|^{2}\right)$

$\geq\left(\beta^{2} \mu_{1}-4 M(v)\right)\left(\|u\|^{2}+\|v\|^{2}\right)$

$\geq\left(\beta^{2} \mu_{1}-4 m_{1}\right)\left(\|u\|^{2}+\|v\|^{2}\right)$.

Since $\beta^{2} \mu_{1}>4 m_{1}, m_{0} \leq M(s) \leq m_{1}$ holds, we can know $\Phi(U, U) \geq 0$. Therefore, for all $U \in X_{N}$, analogously, for all $U \in X_{R}$, we can get

$$
\begin{aligned}
\psi & (U, U) \\
= & \left(\nabla^{m} u, \nabla^{m} u\right)+\left(\nabla^{m} v, \nabla^{m} v\right)+2\left(\nabla^{-m} p, \nabla^{m} u\right)+2\left(\nabla^{-m} q, \nabla^{m} v\right) \\
& +\left(\nabla^{-m} p, \nabla^{-m} p\right)+\left(\nabla^{-m} q, \nabla^{-m} q\right)-4 M(v)(\bar{u}, u) \\
& +2 \beta^{2}\left(\nabla^{m} \bar{u}, \nabla^{m} u\right)-4 M(v)(\bar{v}, v)+2 \beta^{2}\left(\nabla^{m} \bar{v}, \nabla^{m} v\right) \\
= & \left\|\nabla^{m} u\right\|^{2}+\left\|\nabla^{m} v\right\|^{2}+2\left(\nabla^{-m} p, \nabla^{m} u\right)+2\left(\nabla^{-m} q, \nabla^{m} v\right)+\left\|\nabla^{-m} p\right\|^{2} \\
& +\left\|\nabla^{-m} q\right\|^{2}-4 M(v)\|u\|^{2}+2 \beta^{2}\left\|\nabla^{m} u\right\|^{2}-4 M(v)\|v\|^{2}+2 \beta^{2}\left\|\nabla^{m} v\right\|^{2} \\
\geq & \left(2 \beta^{2} \mu_{1}-4 M(v)\right)\left(\|u\|^{2}+\|v\|^{2}\right) \\
\geq & \left(2 \beta^{2} \mu_{1}-4 m_{1}\right)\left(\|u\|^{2}+\|v\|\right)^{2} \geq 0 .
\end{aligned}
$$


From above, we know that for all $U \in X_{R}$, then $\psi(U, U) \geq 0$ holds. So, we define a scale product with $\Phi$ and $\psi$ in $X$.

$$
\langle\langle U, V\rangle\rangle_{X}=\Phi\left(P_{N} U, P_{N} V\right)+\psi\left(P_{R} U, P_{R} V\right),
$$

where $P_{N}, P_{R}$ are respectively the projection: $X \rightarrow X_{N}, X \rightarrow X_{R}$.

In the inner product of $X$ in (3.29), $X_{1}$ and $X_{2}$ are orthogonal. In fact, we need prove that $X_{1}$ and $X_{c}$ are orthogonal.

$$
\begin{aligned}
\left\langle\left\langle U_{j}^{-}, U_{j}^{+}\right\rangle\right\rangle_{X}=\Phi\left(U_{j}^{-}, U_{j}^{+}\right) \\
=\beta\left(\nabla^{m} u_{j}, \nabla^{m} \bar{u}_{j}\right)+2 \beta\left(-\lambda_{j}^{+} \nabla^{-m} u_{j}, \nabla^{m} u_{j}\right)+\beta\left(\nabla^{m} v_{j}, \nabla^{m} \bar{v}_{j}\right) \\
+2 \beta\left(-\lambda_{j}^{+} \nabla^{-m} v_{j}, \nabla^{m} v_{j}\right)+2 \beta\left(-\lambda_{j}^{-} \nabla^{-m} u_{j}, \nabla^{m} \bar{u}_{j}\right)+4\left(-\lambda_{j}^{+} \nabla^{-m} u_{j},-\lambda_{j}^{-} \nabla^{-m} u_{j}\right) \\
+2 \beta\left(-\lambda_{j}^{-} \nabla^{-m} v_{j}, \nabla^{-m} \bar{v}_{j}\right)+4\left(-\lambda_{j}^{+} \nabla^{-m} v_{j},-\lambda_{j}^{-} \nabla^{-m} v_{j}\right)-4 M\left(\sqrt[m]{\mu_{j}}\right)\left(\bar{u}_{j}, u_{j}\right) \\
+\left(2 \beta^{2}-\beta\right)\left(\nabla^{m} \bar{u}_{j}, \nabla^{m} u_{j}\right)-4 M\left(\sqrt[m]{\mu_{j}}\right)\left(\bar{v}_{j}, v_{j}\right)+\left(2 \beta^{2}-\beta\right)\left(\nabla^{m} \bar{v}_{j}, \nabla^{m} v_{j}\right) \\
=\beta\left\|\nabla^{m} u_{j}\right\|^{2}+\beta\left\|\nabla^{m} v_{j}\right\|^{2}-2 \beta\left(\lambda_{j}^{+}+\lambda_{j}^{-}\right)\left\|u_{j}\right\|^{2} \\
\quad-2 \beta\left(\lambda_{j}^{+}+\lambda_{j}^{-}\right)\left\|v_{j}\right\|^{2}+4 \lambda_{j}^{-} \lambda_{j}^{+}\left(\left\|\nabla^{-m} u_{j}\right\|^{2}+\left\|\nabla^{-m} v_{j}\right\|^{2}\right)-4 M\left(\sqrt[m]{\mu_{j}}\right)\left\|u_{j}\right\|^{2} \\
+\left(2 \beta^{2}-\beta\right)\left\|\nabla^{m} u_{j}\right\|^{2}-4 M\left(\sqrt[m]{\mu_{j}}\right)\left\|v_{j}\right\|^{2}+\left(2 \beta^{2}-\beta\right)\left\|\nabla^{m} v_{j}\right\|^{2} \\
=2 \beta^{2} \mu_{j}-2 \beta\left(\lambda_{j}^{+}+\lambda_{j}^{-}\right)+4 \lambda_{j}^{-} \lambda_{j}^{+} \frac{1}{\mu_{j}}-4 M\left(\sqrt[m]{\mu_{j}}\right)=0 .
\end{aligned}
$$

According to $\left\|u_{j}\right\|^{2}+\left\|v_{j}\right\|^{2}=1,\left\|\nabla^{m} u_{j}\right\|^{2}+\left\|\nabla^{m} v_{j}\right\|^{2}=\mu_{j}$, $\left\|\nabla^{-m} u_{j}\right\|^{2}+\left\|\nabla^{-m} v_{j}\right\|^{2}=\frac{1}{\mu_{j}}$ and $\lambda_{j}^{+}+\lambda_{j}^{-}=\beta \mu_{j}, \quad \lambda_{j}^{+} \lambda_{j}^{-}=M\left(\sqrt[m]{\mu_{j}}\right) \mu_{j}$, we can get the above results.

Step 3. Next, we estimate the Lipschitz constant $l_{F}$ of $F$,

$$
F(U)=\left(0,0, f_{1}(x)-g_{1}(u, v), f_{2}(x)-g_{2}(u, v)\right)^{\mathrm{T}} .
$$

$g_{i}: H^{m} \times H^{m} \rightarrow H^{m} \times H^{m},(i=1,2)$ are globally Lipschitz continuous with maximum Lipschitz constant $\frac{l}{2}$ of $g_{i}$ from (3.27), (3.28), for arbitrarily $U=(u, v, p, q) \in X$, we have.

Let $P_{1}: X \rightarrow X_{1}, P_{2}: X \rightarrow X_{2}$ are the orthogonal projection.

If $U=(u, v, p, q) \in X, U_{1}=\left(u_{1}, v_{1}, p_{1}, q_{1}\right)=P_{1} U, U_{2}=\left(u_{2}, v_{2}, p_{2}, q_{2}\right)=P_{2} U$ hold, then $P_{1} u=u_{1}, P_{1} v=v_{1}, P_{2} u=u_{2}, P_{2} v=v_{2}$.

$$
\begin{aligned}
\|U\|_{X}^{2} & =\Phi\left(P_{1} U, P_{1} U\right)+\Psi\left(P_{2} U, P_{2} U\right) \\
& \geq\left(\beta^{2} \mu_{1}-4 M\left(v_{1}\right)\right)\left(\left\|u_{1}\right\|^{2}+\left\|v_{1}\right\|^{2}\right)+\left(2 \beta^{2} \mu_{1}-4 M\left(v_{2}\right)\right)\left(\left\|u_{2}\right\|^{2}+\left\|v_{2}\right\|^{2}\right) \\
& \geq\left(\beta^{2} \mu_{1}-4 m_{1}\right)\left(\|u\|^{2}+\|v\|^{2}\right)
\end{aligned}
$$

Let $U=\left(u_{1}, v_{1}, \bar{u}_{1}, \bar{v}_{1}\right), V=\left(u_{2}, v_{2}, \bar{u}_{2}, \bar{v}_{2}\right)$, then 


$$
\begin{aligned}
& \|F(U)-F(V)\|_{X} \\
& =\left\|g_{1}\left(u_{1}, v_{1}\right)-g_{1}\left(u_{2}, v_{2}\right)\right\|_{H_{0}^{m} \times H_{0}^{m}}+\left\|g_{2}\left(u_{1}, v_{1}\right)-g_{2}\left(u_{2}, v_{2}\right)\right\|_{H_{0}^{m} \times H_{0}^{m}} \\
& \leq \frac{l}{2}\left(\left\|u_{1}-u_{2}\right\|_{H_{0}^{m}}+\left\|v_{1}-v_{2}\right\|_{H_{0}^{m}}\right)+\frac{l}{2}\left(\left\|u_{1}-u_{2}\right\|_{H_{0}^{m}}+\left\|v_{1}-v_{2}\right\|_{H_{0}^{m}}\right) \\
& =l\left(\left\|u_{1}-u_{2}\right\|_{H_{0}^{m}}+\left\|v_{1}-v_{2}\right\|_{H_{0}^{m}}\right) \leq \frac{\sqrt{2} l}{\sqrt{\beta^{2} \mu_{1}-4 m_{1}}}\|U-V\|_{X} .
\end{aligned}
$$

Therefore $l_{F} \leq \frac{\sqrt{2} l}{\sqrt{\beta^{2} \mu_{1}-4 m_{1}}}$.

Step 4. Now, we need prove the spectral gap condition (2.6) holds.

From the above mentioned $\Lambda_{1}=\lambda_{N}^{-}$and $\Lambda_{2}=\lambda_{N+1}^{-}$, we can get

$$
\Lambda_{2}-\Lambda_{1}=\lambda_{N+1}^{-}-\lambda_{N}^{-}=\frac{\beta}{2}\left(\mu_{N+1}-\mu_{N}\right)+\frac{1}{2}(\sqrt{R(N)}-\sqrt{R(N+1)}),
$$

where $R(N)=\beta^{2} \mu_{N}^{2}-4 \mu_{N} M\left(\sqrt[m]{\mu_{N}}\right)$.

We determine $N_{1}>0$ such that for all $N \geq N_{1}$, let

$$
R_{1}(N)=1-\sqrt{\frac{\beta^{2}}{\beta^{2} \mu_{1}-4 m_{1}}-\frac{4 m_{1}}{\mu_{N}\left(\beta^{2} \mu_{1}-4 m_{1}\right)}},
$$

we can get

$$
\begin{aligned}
& \sqrt{R(N)}-\sqrt{R(N+1)}+\sqrt{\beta^{2} \mu_{1}-4 m_{1}}\left(\mu_{N+1}-\mu_{N}\right) \\
& =\sqrt{\beta^{2} \mu_{1}-4 m_{1}}\left(\mu_{N+1} R_{1}(N+1)-\mu_{N} R_{1}(N)\right) .
\end{aligned}
$$

According to the previous hypothesis $\varepsilon<m_{0} \leq M(s) \leq m_{1}=\frac{\beta^{2} \mu_{1}-1}{4}$, we can know

$$
\lim _{N \rightarrow \infty}\left(\sqrt{R(N)}-\sqrt{R(N+1)}+\sqrt{\beta^{2} \mu_{1}-4 m_{1}}\left(\mu_{N+1}-\mu_{N}\right)\right)=0 .
$$

Then, combining (3.33), (3.34), (3.17) and (3.37), we obtain

$$
\begin{aligned}
& \Lambda_{2}-\Lambda_{1}>\left(\mu_{N+1}-\mu_{N}\right)\left(\frac{\beta}{2}-\frac{1}{2} \sqrt{\beta^{2} \mu_{1}-4 m_{1}}\right)-1 \\
& \geq \frac{4 \sqrt{2} l}{\sqrt{\beta^{2} \mu_{1}-4 m_{1}}} \geq 4 l_{F} .
\end{aligned}
$$

The proof is completed.

Theorem 3.2. [8] Under the condition of Theorem 3.1, the initial boundary value problem (1.1)-(1.6) admits an inertial manifold $\mu$ in $X$ of the form

$$
\mu=\operatorname{graph}(\rho)=\left\{\omega+\rho(\zeta): \zeta \in X_{1}\right\},
$$

where $X_{1}, X_{2}$ are as in (3.20), (3.21) and $\rho: X_{1} \rightarrow X_{2}$ is a Lipschitz continuous function.

\section{Conclusion}

In this paper, we prove the existence of the inertial manifolds for a class of high- 
er-order coupled Kirchhoff-type equations. In the process of research, we take advantage of Hadamard's graph to get the equivalent form of the original equations and then use spectral gap condition. Based on some of the work above, we prove the existence of the inertial manifolds of the system. For this problem, we will study the exponential attractors, blow-up, random attractors and so on.

\section{Acknowledgements}

We express our heartful thanks to the anonymous reader for his/her careful reading of this paper. We hope that we can obtain valuable comments and advices. These contributions vastly improved the paper and making the paper better.

\section{References}

[1] Wu, J.Z. and Lin, G.G. (2010) An Inertial Manifold of the Two-Dimensional Strongly Damped Boussinesq Equation. Journal of Yunnan University (Natural Science Edition), 32, 119-224.

[2] Xu, G.G., Wang, L.B. and Lin, G.G. (2014) Inertial Manifold for a Class of the Retarded Nonlinear Wave Equations. Mathematica Applicata, 27, 887-891.

[3] Lou, R.J., Lv, P.H. and Lin, G.G. (2016) Exponential Attractors and Inertial Manifolds for a Class of Generalized Nonlinear Kirchhoff-Sine-Gordon Equation. Journal of Advances in Mathematics, 12, 6361-6375.

[4] Chen, L., Wang, W. and Lin, G.G. (2016) Exponential Attractors and Inertial Manifolds for the Higher-Order Nonlinear Kirchhof-Type Equation. International Journal of Modern Communication Technologies \& Research, 4, 6-12.

[5] Lin, G.G. (2011) Nonlinear Evolution Equation. Yunnan University Press, Kunming.

[6] Robinson, J.C. (2001) Infinite Dimensional Dynamical System. Cambridge University Press, London.

[7] Zheng, S.M. and Milani, A. (2004) Exponential Attractors and Inertial Manifold for Singular Perturbations of the Cahn-Hilliard Equations. Nonlinear Analysis, 57, 843-877. https://doi.org/10.1016/j.na.2004.03.023

[8] Tmam, R. (1988) Infinite Dimensional Dynamical Systems in Mechanics and Physics. Springer Verlag, New York. https://doi.org/10.1007/978-1-4684-0313-8 